\title{
MANIFOLDS: HAUSDORFFNESS VERSUS HOMOGENEITY
}

\author{
MATHIEU BAILLIF AND ALEXANDRE GABARD \\ (Communicated by Alexander N. Dranishnikov)
}

\begin{abstract}
We analyze the relationship between Hausdorffness and homogeneity in the frame of manifolds not confined to be Hausdorff. We exhibit examples of homogeneous non-Hausdorff manifolds and prove that a Lindelöf homogeneous manifold is Hausdorff.
\end{abstract}

\section{INTRODUCTION}

Our purpose here is to analyze the relationship between Hausdorffness and homogeneity in the frame of manifolds. We give the word manifold its broadest sense, that is, a topological space locally homeomorphic to the Euclidean space $\mathbb{R}^{n}$ of a fixed dimension (without assuming the Hausdorff separation axiom).

Recall that a connected Hausdorff manifold $M$ is homogeneous, i.e. for each $x, y \in M$, there is a homeomorphism $h: M \rightarrow M$ taking $x$ to $y$ (see [9] or [10, p. 150]).

This property is true only under the Hausdorff assumption. Without it, one may well have a nonhomogeneous manifold, for example the well-known line with two origins: take two copies of the real line $\mathbb{R}$ and identify all corresponding points of the copies but the origin (Figure 1). This yields a one-dimensional manifold in which the two origins cannot be separated. ${ }^{1}$ Notice though that a point different from the origins can be separated from any other point, so the manifold is not homogeneous. Another well-known example of a non-Hausdorff manifold is the branching line obtained by identifying the points $<0$ in the two copies of $\mathbb{R}$ (Figure 1 ).

One may think that homogeneity is a sufficient condition to characterize, in the realm of manifolds, those which are Hausdorff. We show that this is not the case by exhibiting two examples. The first, called the complete feather or everywhere branching line $F$ will be discussed in $\S 2$. It was first defined by Haefliger and Reeb in [4] and is constructed by "grafting" lines to all points of a line and iterating this process indefinitely (see Figure 2).

Here $F$ is a non-Hausdorff homogeneous 1-manifold but it is neither separable ${ }^{2}$ nor Lindelöf. ${ }^{3}$ It furthermore has some interesting contractibility properties: it is contractible but not strongly (i.e. in such a way that the collapsing point stays fixed

Received by the editors September 5, 2006 and, in revised form, November 1, 2006.

2000 Mathematics Subject Classification. Primary 57N99, 54D10, 54E52.

${ }^{1}$ We say that two points of a topological space can be separated if there are two disjoint open sets containing one of them each.

${ }^{2} \mathrm{~A}$ space is separable if it has a countable dense subspace.

${ }^{3} \mathrm{~A}$ space is Lindelöf if each open cover has a countable subcover. 

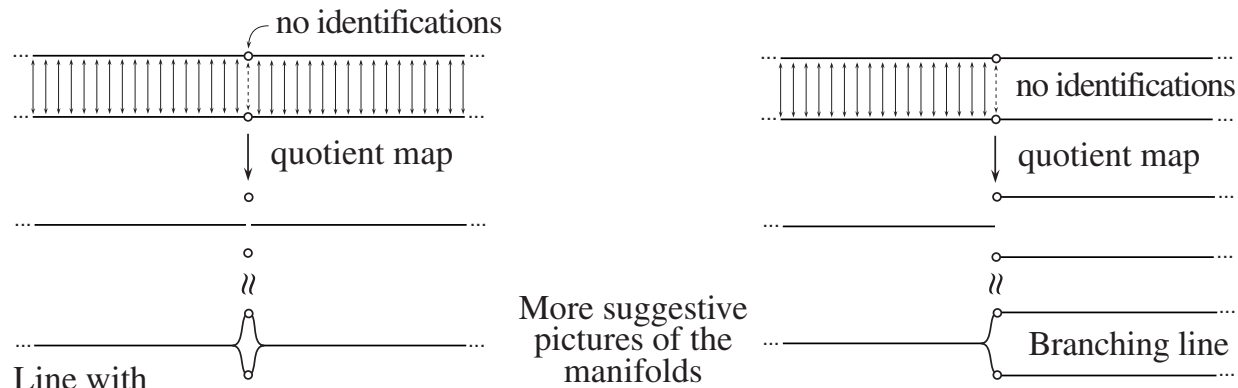
two origins

FIGURE 1. Classical constructions of non-Hausdorff manifolds.

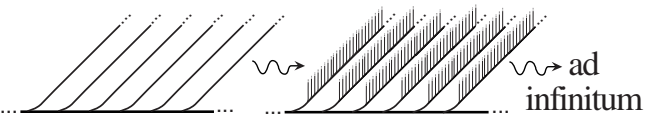

FIGURE 2. Haefliger-Reeb's construction of the "plume complète" (here called complete feather).

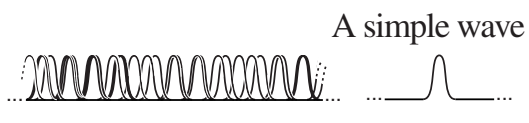

FIGURE 3. Another example of homogeneous non-Hausdorff manifold.

during the deformation), even though each point has such a strongly contractible neighborhood. This answers a question left open in [3].

Our second example is the everywhere doubled line D, a "continuous" version of the line with two origins in which we perform the duplication process at all points (Figure 3 tries to give a representation). The line $D$ is homogeneous, separable but neither Hausdorff nor Lindelöf. A discussion of this example is the object of $\S 3$. The quest for a Lindelöf example is ruled out by the following:

Theorem 1.1. A homogeneous Lindelöf manifold is Hausdorff (and therefore is metrizable). ${ }^{4}$

This will be proved in $\S 4$. The proof uses that a manifold is a Baire space ${ }^{5}$ (since a space that is locally Baire ${ }^{6}$ is in fact Baire). Alternatively we can also argue that a (not necessarily Hausdorff) locally compact space is Baire, a fact that is usually proved only for Hausdorff spaces (see for instance [1]) but which remains valid in this more general setting (see $\S 5$ ).

\section{The COMPLETE FEATHer $F$}

Let us first give a loose description of $F$. The idea is to start with the usual real line and to add branches (as in the branching line) at any $x \in \mathbb{R}$. This results in a "hairy line", with branches at level 1 . Then, we continue the process by adding new branches at level 2 to all points in branches in level 1, and so on indefinitely. The resulting space $F$ is a 1-manifold whose homogeneity comes from the fact that we can "flip" a branch at level $i$ with a branch at level $i+1$. It is also contractible.

\footnotetext{
${ }^{4}$ Recall that a Lindelöf Hausdorff manifold is metrizable; this follows from Urysohn's metrization theorem, since Lindelöf and locally second countable imply second countable.

${ }^{5}$ Every countable intersection of dense open sets is dense.

${ }^{6}$ For any property $\mathcal{P}$ attributable to a space, a space is said to be locally $\mathcal{P}$ if each of its points has a neighborhood with the property $\mathcal{P}$.
} 

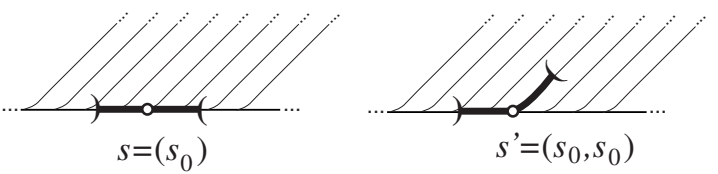

FIGURE 4. Typical neighborhoods of points according as the point is down or up.

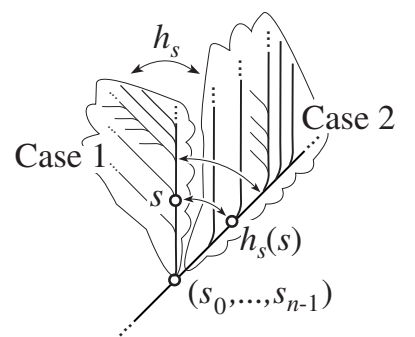

FIGURE 5. Proving homogeneity.

Now we give the formal definition. The underlying set of $F$ is

$$
\left\{\begin{array}{c}
s=\left(s_{0}, \ldots, s_{n}\right) \text { for some } n \geq 0, s_{i} \in \mathbb{R}, \\
\text { and } s_{0}<s_{1}<\cdots<s_{n-1} \leq s_{n}
\end{array}\right\} .
$$

Notice that the last inequality is not strict. One should interpret the sequences of length 1 as the usual real line, those of length $\leq 2$ as the hairy line, and so on. In fact, the first $n$ entries of a sequence $\left(s_{0}, \ldots, s_{n}\right)$ indicate positions where branching has taken place while the last entry parameterizes the "remainder" of the branch. We topologize $F$ with the order topology for the following partial order:

$$
\left(s_{0}, \ldots, s_{n}\right)<\left(t_{0}, \ldots, t_{m}\right) \text { iff } n \leq m, s_{i}=t_{i} \text { for } i=0, \ldots, n-1 \text { and } s_{n}<t_{n} .
$$

Notice that $\left(s_{0}, \ldots, s_{n}\right)$ and $\left(s_{0}, \ldots, s_{n}, s_{n}\right)$ are incomparable and have the same predecessors. The feather $F$ is a "tree" in the sense that the predecessors of any point are totally ordered.

Proposition 2.1. F is a connected homogeneous non-Hausdorff 1-manifold.

Notice that $F$ is nonseparable. In fact there is even an uncountable family of pairwise disjoint open sets in $F$.

Proof. One sees immediately that $F$ is a non-Hausdorff manifold, since the intervals are homeomorphic to $\mathbb{R}$ and the points of the form $\left(s_{0}, \ldots, s_{n}\right)$ and $\left(s_{0}, \ldots, s_{n}, s_{n}\right)$ cannot be separated. Connectedness is also easy. To see that $F$ is homogeneous, we first show that given any point $s=\left(s_{0}, \ldots, s_{n}\right) \in F$, there is a homeomorphism of $F$ sending $s$ to $\left(s_{n}\right) \in F$. We first consider the map $h_{s}: F \rightarrow F$ that flips the two "branches" emanating from $\left(s_{0}, \ldots, s_{n-1}\right)$ (see Figure 5$)$. It is given by the formula

$h_{s}(r)=\left\{\begin{array}{cl}\left(s_{0}, \ldots, s_{n-2}, r_{n}, r_{n+1}, \ldots, r_{m}\right) & \text { if } r=\left(s_{0}, \ldots, s_{n-2}, s_{n-1}, r_{n}, r_{n+1}, \ldots, r_{m}\right), \\ \left(s_{0}, \ldots, s_{n-2}, s_{n-1}, r_{n-1}, r_{n}, \ldots, r_{m}\right) & \text { if } r=\left(s_{0}, \ldots, s_{n-2}, r_{n-1}, r_{n}, \ldots, r_{m}\right) \text { and } r_{n-1}>s_{n-1}, \\ \left(s_{0}, \ldots, s_{n-2}, s_{n-1}, s_{n-1}\right) & \text { if } r=\left(s_{0}, \ldots, s_{n-2}, s_{n-1}\right), \\ r & \text { otherwise. }\end{array}\right.$

One sees easily that $h_{s}$ is a homeomorphism (actually it is an involution). For $s=\left(s_{0}, \ldots, s_{n}\right) \in F$ and $k \leq n$, let $s^{(k)}=\left(s_{0}, \ldots, s_{n-k}\right)$. Then, $h_{s^{(n-1)}} \circ \cdots \circ$ $h_{s^{(1)}} \circ h_{s}(s)=\left(s_{n}\right)$. To finish the proof, it suffices to remark that for $t \in \mathbb{R}$ the map $\left(s_{0}, \ldots, s_{n}\right) \mapsto\left(s_{0}+t, \ldots, s_{n}+t\right)$ is a homeomorphism. 
Lemma 2.2. $F$ is contractible.

Proof. The idea is to contract all points of the form $\left(s_{0}, \ldots, s_{n-1}, s_{n}\right)$ on the point $\left(s_{0}, \ldots, s_{n-1}, s_{n-1}\right)$ between time $\frac{1}{n+1}$ and $\frac{1}{n}$. For $x \in \mathbb{R}$ and $t \in[0,1]$, let $\varphi_{t}^{x}$ : $\mathbb{R} \rightarrow \mathbb{R}$ be defined by

$$
\varphi_{t}^{x}(y)=\left\{\begin{array}{cl}
y & \text { if } y<x \\
(1-t) y+t x & \text { if } y \geq x
\end{array}\right.
$$

Then, $\varphi_{0}^{x}=\mathrm{id}$, and $\varphi_{1}^{x}(y)=x$ for all $y \geq x$. If $s=\left(s_{0}, \ldots, s_{n}\right) \in F$, we let $\phi_{t}(s)=$ $\left(s_{0}, \ldots, s_{n-1}, \varphi_{t}^{s_{n-1}}\left(s_{n}\right)\right)$, so $\phi_{1}(s)=\left(s_{0}, \ldots, s_{n-1}, s_{n-1}\right)$. If $s=\left(s_{0}, \ldots, s_{n}\right)$, we write $t^{\prime}=n(n+1) t-n$ and define

$$
h_{t}(s)=\left\{\begin{array}{cl}
s & \text { if } n=0 \text { or } t \leq \frac{1}{n+1}, \\
\phi_{t^{\prime}}(s) & \text { if } n \neq 0 \text { and } t \in\left[\frac{1}{n+1}, \frac{1}{n}\right], \\
h_{t}\left(\left(s_{0}, \ldots, s_{n-1}\right)\right) & \text { if } n \neq 0 \text { and } t>\frac{1}{n} .
\end{array}\right.
$$

The definition is implicit, but this causes no problem: we proceed by induction on $n$. Thus, $h_{0}=\mathrm{id}$ and $h_{1}(s)=\left(s_{0}, s_{0}\right)$ if $s=\left(s_{0}, \ldots, s_{n}\right)$ with $n>0, h_{1}\left(\left(s_{0}\right)\right)=\left(s_{0}\right)$. We then define $h_{t}\left(\left(s_{0}\right)\right)=h_{t}\left(\left(s_{0}, s_{0}\right)\right)=\left(s_{0}-t+1\right)$ for $\left.\left.t \in\right] 1,2\right]$. It is not difficult to see that $h_{t}$ is continuous and that $h_{2}(F)$ is included in the sequences of length 1 which are homeomorphic to $\mathbb{R}$ and thus contractible.

A space $X$ is strongly contractible to the point $p$ if there exists a homotopy $h_{t}: X \rightarrow X$ such that $h_{0}=\mathrm{id}, h_{1} \equiv p$ and $h_{t}(p)=p$ for all $t$. D. Gauld [3] showed that if $X$ is contractible, locally strongly contractible to $p$ and completely regular at $p$, then $X$ is strongly contractible to $p$. Further he asked whether "completely regular" could be dropped; the complete feather $F$ gives a counterexample since:

Lemma 2.3. $F$ is not strongly contractible to any of its points.

Proof. Call twins the pairs of points of $F$ of the form $\left\{\left(s_{0}, \ldots, s_{n}\right),\left(s_{0}, \ldots, s_{n}, s_{n}\right)\right\}$. Any sequence $\left(s_{0}, \ldots, s_{n-1}, s_{n}^{m}\right)(m \in \mathbb{N})$ with $s_{n}^{m} \nearrow s_{n}$ converges to both twins. Thus, if one of the twins moves, the other must also move. Since any point of $F$ has a twin, the result follows.

\section{THE EVERYWHERE DOUBLED Line $D$}

We can build $D$ either as an inductive limit or with two copies of the line with an exotic topology. We give the latter construction. The underlying set of $D$ is $\mathbb{R} \times\{0,1\}$. Points of $D$ with zero second coordinate are called "down", the others "up". A base for the topology is given by usual open sets downstairs with a finite (eventually zero) number of points removed and lifted upstairs, that is, subsets of the form

$$
\mathcal{U}_{O, F}=(O \backslash F) \times\{0\} \cup F \times\{1\},
$$

where $O \subset \mathbb{R}$ is open and $F \subset O$ finite. Such subsets will be called waves. It is immediate that the waves are closed under finite intersections. We topologize $D$ with the topology given by this base.

Proposition 3.1. The everywhere doubled line $D$ is a connected non-Hausdorff homogeneous separable 1-manifold.

Proof. First, it is clear that $D$ is non-Hausdorff, since two points $x, y$ having the same first coordinate cannot be separated. It is also immediate that $\mathbb{Q} \times\{0\}$ is dense in $D$ and that any wave $\mathcal{U}_{O, F}$ where $\left.O=\right] a, b[$ is homeomorphic to $\mathbb{R}$. This proves that $X$ is a separable 1-manifold. Connectedness is also easy. 
To check homogeneity, we begin by observing that the translations $t_{s}:(t, i) \mapsto$ $(t+s, i)$ with $s \in \mathbb{R}$ are clearly homeomorphisms. This settles the case where the two given points have the same second coordinates. If not, use the map exchanging up and down at one value of the abscissae

$$
e_{s}:(t, i) \mapsto \begin{cases}(t, 1-i) & \text { if } t=s, \\ (t, i) & \text { if } t \neq s,\end{cases}
$$

which is a homeomorphism, since it acts simply by adding or removing an oscillation to a given wave (or eventually does nothing at all if $s$ is outside the wave's range).

Note. Working with reflections and exchange maps, we even see that $X$ is involutorially homogeneous (i.e. the homeomorphism taking $x$ to $y$ can always be chosen to be an involution).

Remarks. As a variant of this construction we can also triple each point of the line. This gives a counterexample to an erroneous claim made by Fuks-Rokhlin who asserted that any one-dimensional manifold becomes disconnected after one removes two suitably chosen points (see [2, p. 135]).

It is known that a Hausdorff connected manifold of dimension $\geq 1$ has cardinality the continuum (see Spivak [7] or Nyikos [5]). The same construction as above starting with $\mathbb{R} \times \kappa$ for $\kappa$ any cardinal shows that (homogeneous) connected manifolds can have arbitrarily large cardinality.

\section{Proof of Theorem 1.1}

We will prove the following:

Theorem 4.1. Let $X$ be a topological space which is homogeneous, Lindelöf, locally Hausdorff and Baire. Then $X$ is Hausdorff.

Theorem 1.1 is then immediate. We will need the following application of Zorn's lemma:

Lemma 4.2. Let $X$ be a locally Hausdorff space. Then for each point $x \in X$, there exists a Hausdorff dense open set $U_{x}$ containing $x$.

Proof of Lemma 4.2. Let $x \in X$. Consider $\mathfrak{O}_{x}$ the set of all Hausdorff open sets containing $x$, ordered by inclusion. Since $X$ is locally Hausdorff, $\mathfrak{O}_{x}$ is nonempty. We check that $\mathfrak{O}_{x}$ is inductive. Let $\mathfrak{C}$ be a totally ordered subset of $\mathfrak{O}_{x}$. As usual let $V=\bigcup_{U \in \mathfrak{C}} U$ be the natural upper bound of $\mathfrak{C}$. Then $V$ is open and Hausdorff: given two points in $V$ (say $y, z$ ), each of them belongs to some $V_{y}, V_{z} \in \mathfrak{C}$. But since $\mathfrak{C}$ is totally ordered, both points belong to one of them (say $V_{y}$ ) and can thus be separated by open sets of $V_{y}$, which are also open in $X$.

By Zorn's lemma, there is a $U_{x}$ maximal in $\mathfrak{O}_{x}$. We check its density. So, let $\Omega$ be a nonempty open set of $X$. We can assume that $\Omega$ is Hausdorff. If $\Omega \cap U_{x}=\emptyset$, since $\Omega$ and $U_{x}$ are Hausdorff, so is their union, and thus $U_{x} \cup \Omega \in \mathfrak{O}_{x}$, which contradicts the maximality of $U_{x}$.

Proof of Theorem 4.1. By homogeneity, it is enough to prove the existence of a point $x_{0} \in X$ which can be separated from every other point $y \in X$.

For all $x \in X$ let $U_{x} \ni x$ be given by Lemma 4.2. The collection $\left(U_{x}\right)_{x \in X}$ is an open cover of $X$ from which we extract a countable subcover $\left(U_{x_{i}}\right)_{i \in \mathbb{N}}$ (by 
Lindelöfness). Since the $U_{x_{i}}$ are dense open sets and $X$ is Baire, their intersection $\bigcap_{i \in \mathbb{N}} U_{x_{i}}$ is dense and so in particular nonempty. Any point $x_{0}$ in this intersection is separable from any other $y \in X$ : since the $\left(U_{x_{i}}\right)_{i \in \mathbb{N}}$ cover $X, y$ is in $U_{x_{i}}$ for some $i \in \mathbb{N}$; but so does $x_{0}$, and since $U_{x_{i}}$ is Hausdorff, $x_{0}$ and $y$ can be separated.

Remarks. The preceding results raised some problems we found worth mentioning here. Firstly, the Lindelöf condition in Theorem 1.1 is in a sense too strong, since it implies metrizability and there are nonmetrizable Hausdorff manifolds.

Problem 4.3. In Theorem 1.1, can Lindelöf be replaced by a weaker condition in order to ensure the Hausdorffness but not necessarily the metrizability of the manifold?

Secondly, the homogeneous non-Hausdorff manifolds $F$ and $D$ both contain an uncountable (closed) discrete subset: Take one point in each branch at level one in $F$ and all the "up" points in $D$. So, another problem is

Problem 4.4. Is there a homogeneous non-Hausdorff manifold that contains no uncountable (closed) discrete subset, or even stronger, that is hereditarily separable? ${ }^{7}$

(Whether there are nonmetrizable HS Hausdorff manifolds or not is known to be independent of ZFC. Under $C H$, Rudin-Zenor [6] constructed a nonmetrizable $H S$ manifold. On the other hand Szentmiklóssy [8] showed that under $M A+\neg C H$, every locally compact $H S$ Hausdorff space is $H L(=$ hereditarily Lindelöf $)$ and so metrizable if a manifold. The result in [8] is actually stated with compact instead of locally compact, but the above follows by taking the one-point compactification.) A negative answer to the first part of Problem 4.4 would yield that $\omega_{1}$-compact ${ }^{8}$ is an answer to Problem 4.3.

\section{About the Baire Property}

We call a space quasi-compact if from any open cover one may extract a finite subcover, and we call it compact if moreover it is Hausdorff.

Theorem 5.1 (Baire slightly extended). Let X be a locally compact (not necessarily Hausdorff) space. Then $X$ is a Baire space.

The following lemma shows that the classical nesting argument used in the proof of Baire's theorem can be applied to $X$.

Lemma 5.2. Let $X$ be a locally compact space. Then for each $x \in X$ and each neighborhood $V \ni x$ there is a compact neighborhood $U \subset V$ of $x$ ( $X$ is then said to be microcompact).

Proof. Let us denote by $\mathfrak{V}_{x}$ the set of all neighborhoods of $x$. Recall that a compact space is regular, i.e. for each point $x \in X$ and each $V \in \mathfrak{V}_{x}$, there is a closed set $F \in \mathfrak{V}_{x}$ with $F \subset V$. Let $V \in \mathfrak{V}_{x}$, and let $K$ be a compact neighborhood in $\mathfrak{V}_{x}$. Then clearly $V \cap K \in \mathfrak{V}_{x}^{K}$, i.e. is a neighborhood of $x$ in $K$. So, there is $F \in \mathfrak{V}_{x}^{K}$ a closed set of $K$ with $F \subset V \cap K$. So $F$ is compact, contained in $V$ and it is easy to check that $F \in \mathfrak{V}_{x}$.

\footnotetext{
${ }^{7}$ In short it is $H S$, and this means that every subspace is separable.

${ }^{8} \mathrm{~A}$ discrete closed set is at most countable.
} 
Using the classical nesting argument, this implies Theorem 5.1.

Remarks. Locally compact cannot be weakened to locally quasi-compact, since a countably infinite set with the finite complement topology is a quasi-compact space which is not Baire. This space is in fact microquasi-compact, that is, has the property given in Lemma 5.2 with 'compact' replaced by 'quasi-compact'. The following chart summarizes the relations between the local compactness properties and how they stand with respect to Baire (jagged arrows mean "does not imply").

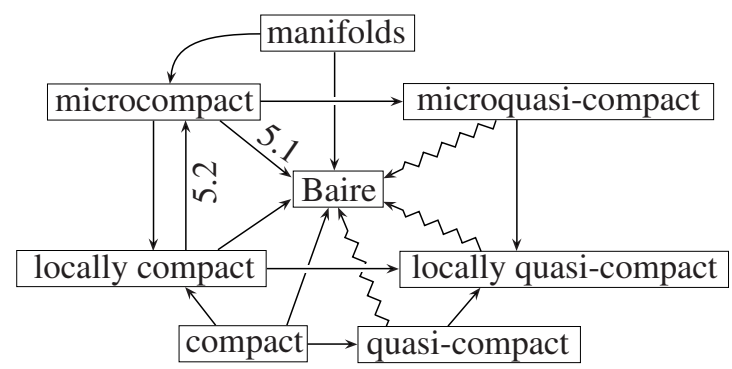

\section{ACKNOWLEDGMENTS}

We thank A. Haefliger and C. Weber for fruitful discussions and the referee for his suggestions.

\section{REFERENCES}

[1] N. Bourbaki. Elements of Mathematics: General Topology, Chapters 1-4, Chapters 5-10 (2nd printing). Springer-Verlag, Berlin, 1989. MR979294 (90a:54001a)

[2] D.B. Fuks and V.A. Rokhlin. Beginner's Course in Topology. Springer-Verlag, Berlin, 1984. MR759162 (86a:57001)

[3] D. Gauld. Strong contractibility. Indian J. Math. 25(1) (1983), 29-32. MR809703 (86k:55005)

[4] A. Haefliger and G. Reeb. Variétés (non séparées) à une dimension et structures feuilletées du plan. Enseignement Math. (2) 3 (1957), 107-125. MR0089412 (19:671c)

[5] P. Nyikos. The theory of nonmetrizable manifolds. In Handbook of Set-Theoretic Topology, 633-684, North-Holland, Amsterdam, 1984. MR776633 (86f:54054)

[6] M.E. Rudin and P. Zenor. A perfectly normal nonmetrizable manifold. Houston J. Math. 2(1) (1976), 129-134. MR0394560 (52:15361)

[7] M. Spivak. Differential Geometry, Vol. 1. Publish or Perish, New York, 1970.

[8] Z. Szentmiklóssy. S-spaces and L-spaces under Martin's axiom. In Á Császár, ed., Topology, Vol. II, Colloq. Math. Soc. J. Bolyai 23, 1139-1145, North-Holland, Amsterdam, 1980. MR588860 (81k:54032)

[9] D. van Dantzig. Ueber topologisch homogene Kontinua. Fund. Math. 15 (1930), 102-125.

[10] W. Vick. Homology Theory: An Introduction to Algebraic Topology (2nd ed.). Graduate texts in mathematics, Springer-Verlag, Berlin, 1994. MR1254439 (94i:55002)

Université de Genève, Section de Mathématiques, 2-4, Rue du Lièvre, CP 64, 1211 Gent̀ve 4, Suisse

E-mail address: baillif@math.unige.ch

Université de Genève, Section de Mathématiques, 2-4, Rue du Lièvre, CP 64, 1211 Gent̀ve 4, Suisse

E-mail address: alexandregabard@hotmail.com 